\title{
Inflammatory macrophages in pancreatic acinar cell metaplasia and initiation of pancreatic cancer
}

\author{
Geou-Yarh Liou ${ }^{1}$ and Peter Storz ${ }^{1}$ \\ ${ }^{1}$ Department of Cancer Biology, Mayo Clinic Comprehensive Cancer Center, Mayo Clinic, Jacksonville, Florida, USA \\ Correspondence to: Peter Storz, email: storz.peter@mayo.edu \\ Keywords: inflammation, pancreatitis, pancreas, acinar-to-ductal metaplasia, macrophages, Kras, pancreatic cancer, PanIN \\ Received: March 6, $2015 \quad$ Accepted: March 26, 2015 \\ Published: March 28, 2015
}

This is an open-access article distributed under the terms of the Creative Commons Attribution License, which permits unrestricted use, distribution, and reproduction in any medium, provided the original author and source are credited.

\section{ABSTRACT:}

The roles of inflammatory macrophages in pancreatic tissue and the development of pancreatic cancer have not been well characterized. Recently it was shown that inflammatory macrophages, besides their function in clearing dead cells, also initiate pancreatic acinar cell metaplasia to duct-like progenitor cells. While in pancreatitis this is a reversible process, in context of an oncogenic stimulus this process is irreversible and can lead to the formation of precancerous lesions. Recent work now indicates that acquisition of an activating Kras mutation in acinar cells initiates signaling that leads to chemoattraction of M1-poliarized macrophages. This oncogene-caused chronic microinflammation can accelerate the pathogenesis of pancreatic cancers.

\section{INTRODUCTION}

Infiltration of macrophages into the pancreas occurs during acute or chronic pancreatitis; and chronic pancreatitis is tightly-linked to development and progression of pancreatic ductal adenocarcinoma (PDA). While the roles of macrophages in pancreatitis are somewhat well established, only little is known on their role in the development of PDA. Differently polarized macrophages (M1 or M2) can exhibit pro- and antiinflammatory properties. M1-polarized macrophages not only remove dead or dying cells via phagocytosis, but are also involved in inflammation-mediated tissue remodeling, while M2-polarized macrophages restrain the inflammatory response and also inhibit the T-cell response [1]. Therefore, for PDA, M2-polarized macrophages have been described as the classical tumor promoting macrophages, while M1-polarized macrophages have been suggested to be tumor preventing. For pancreas biology, the view on M1 macrophages changed with recent publications showing that M1-polarized macrophages can initiate and drive acinar cell transdifferentiation to a duct-like progenitor cell type, a process called acinarto-ductal metaplasia (ADM) [2]. These ADM progenitor cells in context of oncogenic signaling (Kras mutation or augmented EGF-R) initiate the formation of pancreatic lesions and eventually development of PDA [3].
Inflammatory macrophages as drivers of the ADM process in pancreatitis

Acute pancreatitis is a sudden inflammation of the pancreas that can have multiple causes including alcohol abuse, smoking or unhealthy diet. It is driven by damage of pancreatic acinar cells and release of digestive enzymes and pro-inflammatory messengers that lead to macrophage infiltration and activation [4]. During acute pancreatitis pancreatic acinar cells can undergo apoptotic or necrotic cell death or acinar-to-ductal metaplasia. Infiltrated macrophages contribute to clearance of debris and damaged cells, stimulate a further immune response by recruiting T-cells and neutrophils; and often severe the disease to systemic inflammatory response syndrome [5]. However, macrophages also orchestrate the resolution of inflammation by producing anti-inflammatory mediators and may drive processes contributing to pancreas tissue regeneration [6]. For example, acinar-to-ductal metaplasia generates a cellular phenotype that expresses markers for pancreatic progenitor cells. Using animal models and 3D organoid culture of acinar cells, it was demonstrated that during acute pancreatitis macrophages can drive the ADM process [2]. This is mediated through macrophagesecreted cytokines such as tumor necrosis factor (TNF) and chemokines such as CCL5/RANTES [2]. In addition, inflammatory macrophages secrete proteinases such as matrix metalloproteinases (MMPs) that contribute 
to remodeling of the microenvironment, or induce the expression of MMPs in acinar cells that undergo ADM [2, $3,6]$. Overall the interplay between removal of dead cells and debris, alterations in the microenvironment and the generation of progenitor cells (via ADM) - all caused by inflammatory macrophages - may provide the setting for pancreas regeneration after injury (Figure 1).

While rapid production of anti-inflammatory cytokines self-limits acute pancreatitis, the failure to terminate pro-inflammatory signaling prevents regeneration and leads to chronic pancreatitis [7]. In humans and genetic animal models chronic pancreatitis clearly has been identified as a risk factor for the development of precancerous lesions and PDA [8-

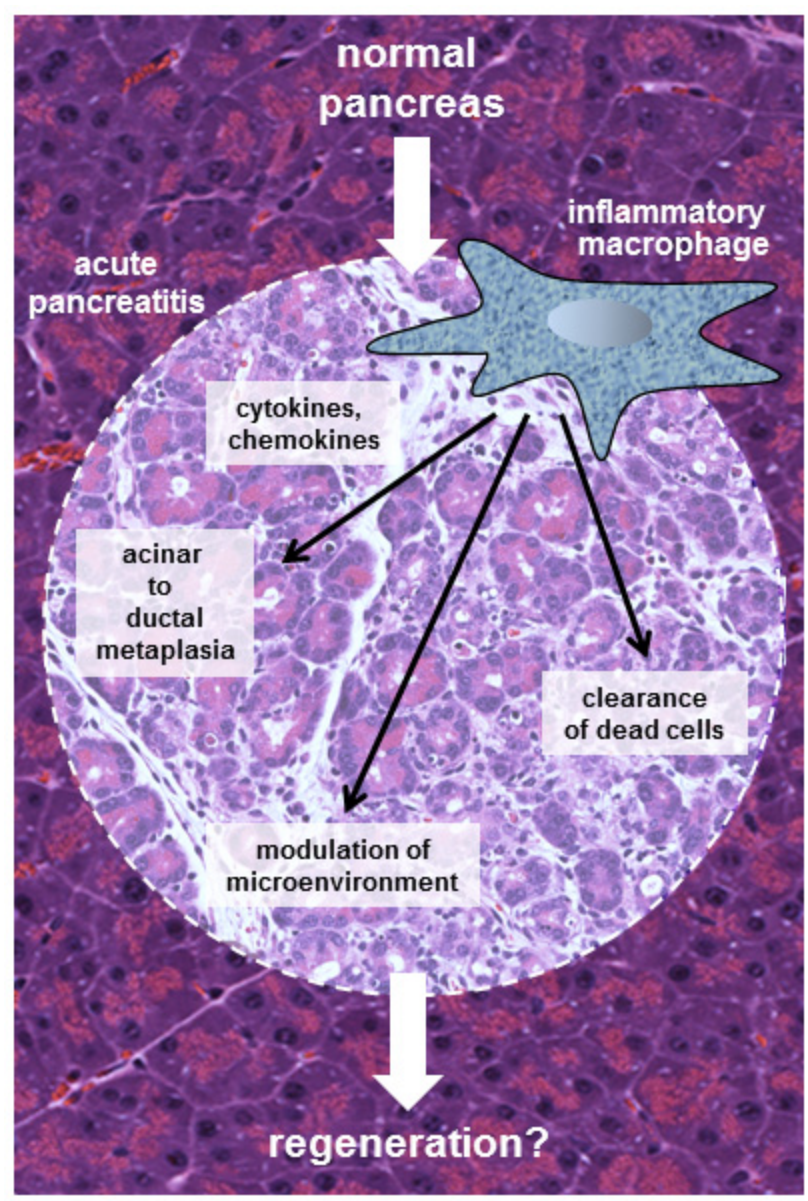

Figure 1: Inflammatory macrophages drive the ADM process during acute pancreatitis. During acute pancreatitis acinar cells can either undergo cell death or a transdifferentiation process named acinar-to-ductal metaplasia (ADM). ADM changes their phenotype to pancreatic progenitorlike cells, which could be important for pancreatic repopulation. Inflammatory macrophages can initiate ADM through release of cytokines and chemokines. They also regulate removal of dead cells and modulation of the microenviroment. The net effect of these different functions of inflammatory macrophages possibly leads to pancreatic regeneration.
11]. This may be explained by the constant presence of macrophages generating an ADM cell type [2, 3], since these progenitor cells in presence of proto-oncogenic signaling (i.e. an activating Kras mutation) can progress to a pre-neoplastic cell type such as the one forming pancreatic intraepithelial neoplasia (PanIN) [12]. Another mechanism of how pancreatitis contributes to development of PDA is by inhibiting Kras-induced senescence [10].

\section{M1-polarized macrophages contribute to the initiation of Kras-caused pre-neoplastic lesions.}

In order to drive the development of pancreatic cancer, oncogenic Kras needs additional inflammatory stimuli to reach pathological activity levels [13, 14]. Even more intriguing is that the acquisition of an oncogenic Kras mutation in acinar cells can initiate microinflammation and chemoattraction of M1-polarized macrophages [3]. This is mediated through induction of expression of intracellular adhesion molecule-1 (ICAM1). ICAM-1 is a surface molecule that can be shed into a soluble form (sICAM-1) [15]. sICAM-1 serves as a chemoattractant for M1-polarized macrophages, while M2-polarized macrophages are not attracted [3]. Attracted macrophages then contribute to metaplasia and formation of pre-neoplastic lesions by secreting proteinases such as matrix-metalloproteinases (MMPs) that facilitate microenvironment remodeling, as well as tumor necrosis factor (TNF) and other inflammatory cytokines (Figure 2 ). The requirement of such crosstalk between acinar cells with Kras mutations and M1-polarized macrophages as a necessary event for the initiation of pancreatic precancerous lesions was demonstrated by depletion of macrophages in animals which attenuated the progression of Kras-caused lesions. Similar effects have been obtained with ICAM-1 neutralizing antibodies [3].

\section{Open Questions and Future Challenges}

Understanding the crosstalk between the pancreatic microenvironment and acinar cells expressing oncogenic mutations is important for several reasons. First, it may lead to discovery of early markers predicting cancer development; and second it could reveal new strategies for intervention.

The finding that M1-polarized macrophages can contribute to tumor initiation is somewhat surprising, since tumor associated macrophages (TAM) often are of a M2polarized phenotype. M1-polarized macrophages so far mainly have been seen as anti-tumorigenic. An immediate question is why M1-polarized macrophages, once attracted to regions of ADM, do not kill precancerous cells? This may be explained by the high antioxidant capacity of pancreatic cells that express an oncogenic version of 
Kras [16]. Macrophages induce cell death of target cells via reactive oxygen species (ROS), and mutant Kras has been shown to upregulate Nrf2, a transcription factor that upregulates a multitude of antioxidant genes [16].

It is also unclear if at some point during initiation or progression of pancreatic lesions signaling is initiated that leads to a switch from M1- to M2-polarized phenotypes [17]. M2-polarized macrophages are needed at a later stage in tumor development to facilitate immunosuppression [18] and angiogenesis [19]. They accelerate lymphatic metastasis and are generally associated with poor prognosis [20]. An open question is if there is a switch from the M1 to the M2 phenotype, or if the two groups are independently recruited.

Eventually, the understanding of the crosstalk between macrophages, pancreatic cells and the pancreatic microenvironment can be important to develop new treatment strategies. Different possibilities arise to target macrophage functions in pancreatitis or developing pancreatic cancer. First, chemoattraction could be blocked using neutralizing antibodies targeting soluble ICAM-1. We have demonstrated that such a strategy is effective in mice to inhibit formation of $\mathrm{Kras}^{\mathrm{G} 12 \mathrm{D}}$-caused PanINs [3]. Similar to our study, mouse monoclonal ICAM-1-blocking antibodies also have been sucessfully-tested in several other animal studies for different disease models in vivo $[21,22]$.

Second, macrophages could be directly targeted. In animal studies, macrophages can be inhibited experimentally with gadolinium chloride [2], liposome encapsulated dichloromethylene-diphosphonate [23], PAF antagonists [24], or macrophage pacifying compounds $[25,26]$ to prevent pancreatitis. In the developing cancer, ablation of macrophages using gadolinium chloride also has been demonstrated to prevent $\mathrm{Kras}^{\mathrm{G} 12 \mathrm{D}}$-mediated microinflammation and formation of precancerous lesions [3]. Other possibilities to deplete macrophage populations in the developing tumor are to block differentiation of hematopoietic stem cells into macrophages by targeting colony stimulating factor 1 (CSF1) or its receptor using chemical compounds or blocking antibodies. In addition, CD40 agonists destruct the tumor stroma by targeting macrophages and also reestablish the tumor immune surveillance in PDA [27].

Macrophages show high plasticity and can change their phenotype and physiology dependent on the microenvironment. Therefore, a third possibility for intervention is to modulate macrophage phenotypes. For example preventing a M1 to M2 subtype switch in pancreata with $\mathrm{Kras}^{\mathrm{G} 12 \mathrm{D}}$-caused lesions, although it may increase ADM events, may also prevent progression to PanINs and pancreatic cancer. A conversion from M1 to M2 can be achieved in vitro after treatment with IL-4, IL10 or IL-13 [28], but it is unclear if this can be achieved in vivo in a pancreas [29].

In summary, to target macrophages or macrophage populations in the developing pancreatic cancer for therapeutic applications requires a full understanding of the phenotypes involved, how they crosstalk with other pancreatic or infiltrated cells, and how this crosstalk contributes to the formation and progression of preneoplastic lesions.

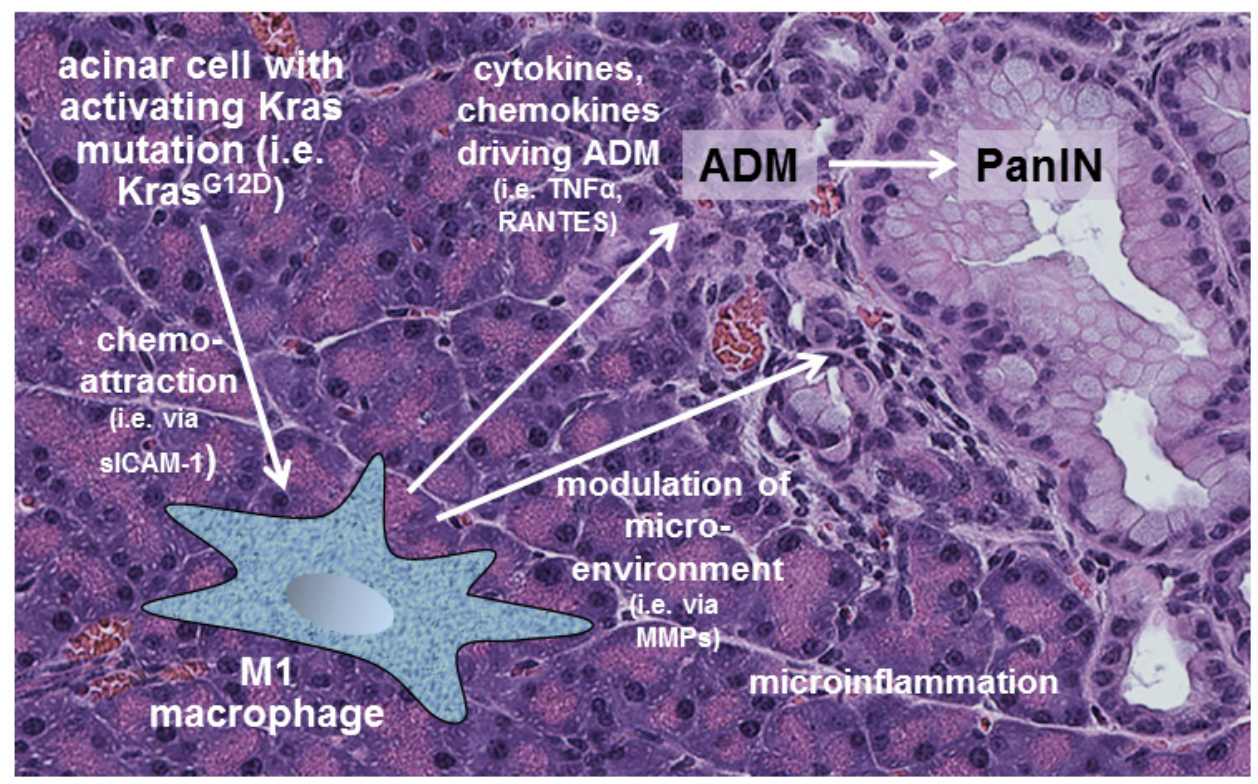

Figure 2: M1-polarized macrophages contribute to the initiation of pre-neoplastic lesions. Acinar cells that acquire an activating Kras mutation (i.e. Kras ${ }^{\mathrm{G} 12 \mathrm{D}}$ ) can cause microinflammation by expressing chemoattractants for M1 macrophages. M1 macrophages then drive $\mathrm{ADM}$ and the formation of pre-neoplastic lesions by providing cytokines, chemokines and proteinases that modulate pancreatic microenvironment. 


\section{ACKNOWLEDGEMENTS}

This work was supported by the NIH grants CA135102 and CA140182 to PS; and by a Postdoctoral Fellowship in Regenerative Medicine from the Center for Regenerative Medicine at Mayo Clinic to GYL.

\section{CONFLICT OF INTEREST}

The authors declare that they have no conflicts of interest.

\section{REFERENCES}

1. Hao NB, Lu MH, Fan YH, Cao YL, Zhang ZR and Yang SM. Macrophages in tumor microenvironments and the progression of tumors. Clin Dev Immunol. 2012; 2012:948098.

2. Liou GY, Doppler H, Necela B, Krishna M, Crawford HC, Raimondo M and Storz P. Macrophage-secreted cytokines drive pancreatic acinar-to-ductal metaplasia through NFkappaB and MMPs. J Cell Biol. 2013; 202(3):563-577.

3. Liou GY, Doppler H, Necela B, Edenfield B, Zhang L, Dawson DW and Storz P. Mutant Kras-induced expression of ICAM-1 in pancreatic acinar cells causes attraction of macrophages to expedite the formation of precancerous lesions. Cancer Discov. 2014.

4. Forsmark CE. Management of chronic pancreatitis. Gastroenterology. 2013; 144(6):1282-1291 e1283.

5. Gea-Sorli S and Closa D. Role of macrophages in the progression of acute pancreatitis. World J Gastrointest Pharmacol Ther. 2010; 1(5):107-111.

6. Criscimanna A, Coudriet GM, Gittes GK, Piganelli JD and Esni F. Activated macrophages create lineage-specific microenvironments for pancreatic acinar- and beta-cell regeneration in mice. Gastroenterology. 2014; 147(5):11061118 e1111.

7. Medzhitov R. Origin and physiological roles of inflammation. Nature. 2008; 454(7203):428-435.

8. Greer JB and Whitcomb DC. Inflammation and pancreatic cancer: an evidence-based review. Curr Opin Pharmacol. 2009; 9(4):411-418.

9. Raimondi S, Lowenfels AB, Morselli-Labate AM, Maisonneuve P and Pezzilli R. Pancreatic cancer in chronic pancreatitis; aetiology, incidence, and early detection. Best Pract Res Clin Gastroenterol. 2010; 24(3):349-358.

10. Guerra C, Collado M, Navas C, Schuhmacher AJ, Hernandez-Porras I, Canamero M, Rodriguez-Justo M, Serrano M and Barbacid M. Pancreatitis-induced inflammation contributes to pancreatic cancer by inhibiting oncogene-induced senescence. Cancer Cell. 2011; 19(6):728-739.
11. Guerra C, Schuhmacher AJ, Canamero M, Grippo PJ, Verdaguer L, Perez-Gallego L, Dubus P, Sandgren EP and Barbacid M. Chronic pancreatitis is essential for induction of pancreatic ductal adenocarcinoma by K-Ras oncogenes in adult mice. Cancer Cell. 2007; 11(3):291-302.

12. Stanger BZ and Hebrok M. Control of cell identity in pancreas development and regeneration. Gastroenterology. 2013; 144(6):1170-1179.

13. Daniluk J, Liu Y, Deng D, Chu J, Huang H, Gaiser S, CruzMonserrate Z, Wang H, Ji B and Logsdon CD. An NFkappaB pathway-mediated positive feedback loop amplifies Ras activity to pathological levels in mice. J Clin Invest. 2012; 122(4):1519-1528.

14. Huang H, Daniluk J, Liu Y, Chu J, Li Z, Ji B and Logsdon CD. Oncogenic K-Ras requires activation for enhanced activity. Oncogene. 2014; 33(4):532-535.

15. Xiao X, Mruk DD and Cheng CY. Intercellular adhesion molecules (ICAMs) and spermatogenesis. Hum Reprod Update. 2013; 19(2):167-186.

16. DeNicola GM, Karreth FA, Humpton TJ, Gopinathan A, Wei C, Frese K, Mangal D, Yu KH, Yeo CJ, Calhoun ES, Scrimieri F, Winter JM, Hruban RH, IacobuzioDonahue C, Kern SE, Blair IA, et al. Oncogene-induced Nrf2 transcription promotes ROS detoxification and tumorigenesis. Nature. 2011; 475(7354):106-109.

17. Mosser DM and Edwards JP. Exploring the full spectrum of macrophage activation. Nat Rev Immunol. 2008; 8(12):958969.

18. Momi N, Kaur S, Krishn SR and Batra SK. Discovering the route from inflammation to pancreatic cancer. Minerva Gastroenterol Dietol. 2012; 58(4):283-297.

19. Lin EY, Li JF, Gnatovskiy L, Deng Y, Zhu L, Grzesik DA, Qian H, Xue XN and Pollard JW. Macrophages regulate the angiogenic switch in a mouse model of breast cancer. Cancer Res. 2006; 66(23):11238-11246.

20. Kurahara H, Shinchi H, Mataki Y, Maemura K, Noma H, Kubo F, Sakoda M, Ueno S, Natsugoe S and Takao S. Significance of M2-polarized tumor-associated macrophage in pancreatic cancer. J Surg Res. 2011; 167(2):e211-219.

21. Burns RC, Rivera-Nieves J, Moskaluk CA, Matsumoto $\mathrm{S}$, Cominelli F and Ley K. Antibody blockade of ICAM1 and VCAM-1 ameliorates inflammation in the SAMP-1/ Yit adoptive transfer model of Crohn's disease in mice. Gastroenterology. 2001; 121(6):1428-1436.

22. Iigo Y, Takashi T, Tamatani T, Miyasaka M, Higashida T, Yagita H, Okumura K and Tsukada W. ICAM-1-dependent pathway is critically involved in the pathogenesis of adjuvant arthritis in rats. J Immunol. 1991; 147(12):41674171.

23. Shifrin AL, Chirmule N, Zhang $\mathrm{Y}$ and Raper SE. Macrophage ablation attenuates adenoviral vector-induced pancreatitis. Surgery. 2005; 137(5):545-551. 
24. Wereszczynska-Siemiatkowska U, Dlugosz JW, Siemiatkowski A, Chyczewski L and Gabryelewicz A. Lysosomal activity of pulmonary alveolar macrophages in acute experimental pancreatitis in rats with reference to positive PAF-antagonist (BN 52021) effect. Exp Toxicol Pathol. 2000; 52(2):119-125.

25. Yang J, Denham W, Carter G, Tracey KJ and Norman J. Macrophage pacification reduces rodent pancreatitisinduced hepatocellular injury through down-regulation of hepatic tumor necrosis factor alpha and interleukin-1beta. Hepatology. 1998; 28(5):1282-1288.

26. Yang J, Denham W, Tracey KJ, Wang H, Kramer AA, Salhab KF and Norman J. The physiologic consequences of macrophage pacification during severe acute pancreatitis. Shock. 1998; 10(3):169-175.
27. Beatty GL, Chiorean EG, Fishman MP, Saboury B, Teitelbaum UR, Sun W, Huhn RD, Song W, Li D, Sharp LL, Torigian DA, O'Dwyer PJ and Vonderheide RH. CD40 agonists alter tumor stroma and show efficacy against pancreatic carcinoma in mice and humans. Science. 2011; 331(6024):1612-1616.

28. Stout RD, Jiang C, Matta B, Tietzel I, Watkins SK and Suttles J. Macrophages sequentially change their functional phenotype in response to changes in microenvironmental influences. J Immunol. 2005; 175(1):342-349.

29. Gea-Sorli S and Closa D. In vitro, but not in vivo, reversibility of peritoneal macrophages activation during experimental acute pancreatitis. BMC Immunol. 2009; $10: 42$. 